\title{
Falsely Elevated Acetaminophen Levels in the Setting of Hyperbilirubinemia
}

\author{
Parth J. Parekh, Thomas J. Manser,* \\ Department of Internal Medicine, Eastern Virginia Medical School, Norfolk, VA, United States
}

\section{Accepted on}

June 29th, 2013

\section{DOI Name}

http://dx.doi.org/10.3126/jaim.v2i2.8782

\section{Keywords \\ Analytical Interference, falsely elevated \\ acetaminophen, hyperbilirubinemia}

\section{Citation}

Parth J. Parekh, Thomas J. Manser. Falsely Elevated Acetaminophen Levels in the Setting of Hyperbilirubinemia. Journal of Advances in Internal Medicine 2013;02(02):74-7.

\begin{abstract}
A 51-year-old male with a history of heavy alcohol abuse presented with alcoholic hepatitis and acute renal failure. Although he denied acetaminophen (APAP) ingestion, he was found to have elevated APAP levels that persisted and actually increased despite treatment with $\mathrm{N}$-acetylcysteine. Review of the literature reveals that falsely elevated APAP levels may rarely occur with patients suffering from liver failure and felt to be related to severe hyperbilirubinemia. Interpretation of APAP levels in patients with severe liver disease and hyperbilirubinemia may be difficult and lead to diagnostic and therapeutic confusion.
\end{abstract}

\section{INTRODUCTION}

Patients presenting with elevated transaminases and jaundice are routinely tested for viral, autoimmune and drug etiologies. Acetaminophen (APAP) toxicity is a common and treatable cause of toxic hepatitis and elevated APAP levels in the setting of acute liver injury usually imply that acetaminophen is a primary contributor to the injury. Rare case reports, however, have described elevated levels in the absence of APAP ingestion in patients with hyperbilirubinemia and this appears to be related to the interference of bilirubin with commonly used assays for APAP.

\section{CASE PRESENTATION}

A 51-year-old male with a past history of heavy alcohol abuse ( $1 / 2$ litre of Vodka/day $\times 20$ years) and hypertension presented to the emergency department with jaundice, tremors and visual hallucinations for two and a half weeks. Past medical history was notable for hypertension, prior pancreatitis and unsuccessful alcohol rehabilitation. His only medication was occasional ibuprofen and he adamantly denied acetaminophen use, which family members confirmed. Vital signs showed BP 124/67 mmHg, P 94 BPM, T $95.6 \mathrm{~F}$ and RR 18/min. Exam was remarkable for cutaneous and scleral icterus, asterixis, spider angiomas and ascites. Lab values showed: WBC of $21.6 \mathrm{~K} / \mathrm{ul}$, total bilirubin of $38.6 \mathrm{mg} / \mathrm{dl}$ (direct $>30.0 \mathrm{mg} / \mathrm{dl}$ ), AST of 209 $\mathrm{U} / \mathrm{L}$, ALT of $97 \mathrm{U} / \mathrm{L}$, INR of 3.00, BUN of $55 \mathrm{mg} / \mathrm{dl}$, Creatinine of $5.2 \mathrm{mg} / \mathrm{dl}$ and venous ammonia level of $255 \mathrm{ug} / \mathrm{dl}$. Abdominal CT scan and ultrasound confirmed cirrhosis, fatty liver, ascites and portal hypertension (figures 1). A diagnostic paracentesis excluded SBP. Gastroenterology and nephrology consultations were obtained and recommended treatment for hepatorenal syndrome due to alcoholic hepatitis with Albumin, Octreotide and Midodrine.

\footnotetext{
* Corresponding author

Thomas J. Manser, MD, FACP

Eastern Virginia Medical School - Department of Internal Medicine

825 Fairfax Avenue Hoffheimer Hall - Suite 445

Norfolk, VA 2350

(757) 446-5794

e-mail:mansertj@evms.edu
} 


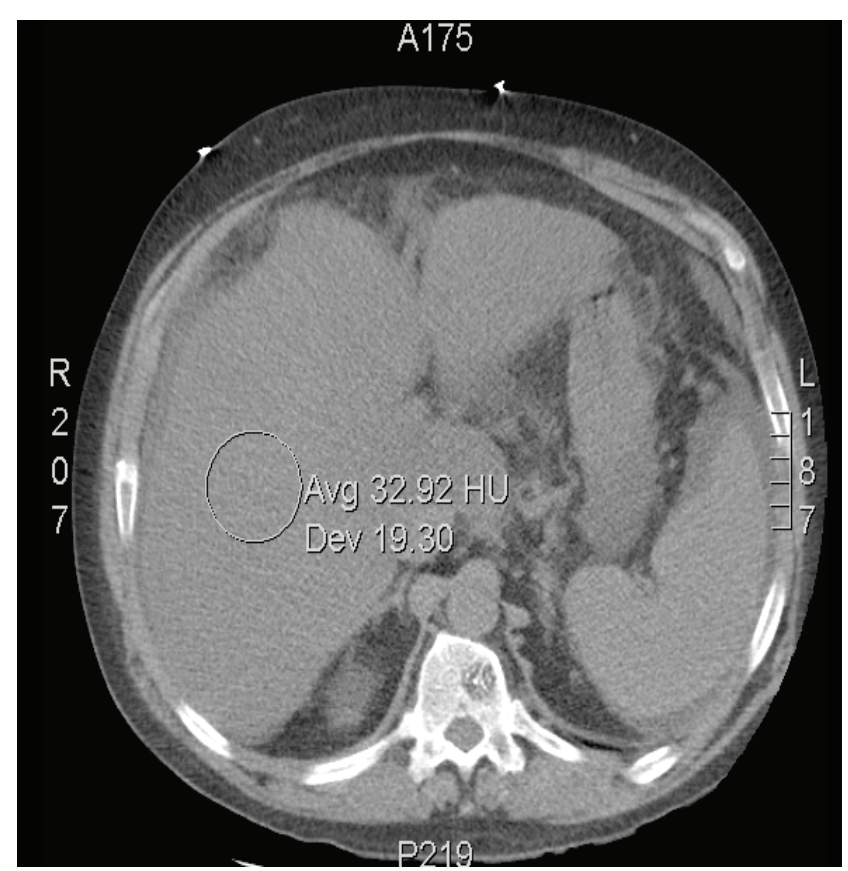

Figure 1: CT abdomen/ Pelvis showing large fatty liver with nodular consistency, splenomegaly and abdominal/ pelvic ascites

The patient was treated with albumin, midodrine, and octreotide for hepatorenal syndrome. Lactulose and and Rifaximin were added for hepatic encephalopathy and propranolol for portal hypertension. Pentoxyfylline and ceftriaxone were briefly added for possible spontaneous bacterial peritonitis (SBP) but stopped after a negative paracentesis. Maddrey's Discriminant Function was calculated at 116 and based on this prednisolone $40 \mathrm{mg} / \mathrm{d}$ was added for severe alcoholic hepatitis.

On day 2 the patient became somnolent, hypoxic and hypotensive and was intubated and transferred to the intensive care unit (ICU) for closer monitoring. At that time an APAP level returned with an initial value of $23 \mathrm{mcg} / \mathrm{ml}$. He was treated for APAP toxicity with N-Acetylcysteine and hepatic function assays and APAP levels were monitored serially. Acetaminophen levels actually rose during subsequent days despite $\mathrm{N}$-Acetylcysteine therapy and were paralleled by increasing serum bilirubin levels. On day 14 the total bilirubin reached a level of $40 \mathrm{mg} / \mathrm{dl}$ at which time the APAP level reached a maximum of $40 \mathrm{mcg} / \mathrm{ml}$.). The patient received a full course of $\mathrm{N}$-acetylcysteine that was discontinued on day 5 . In light of the persistent and increasing APAP levels and given the negative history of ingestion gastroenterology felt the levels were likely spurious and due to the presence of severe hyperbilirubinemia.

The patient was continued on treatment for alcoholic hepatitis and hepatic encephalopathy. He required transient hemodialysis for volume overload. Although he remained markedly jaundiced, azotemic and coagulopathic, he improved globally and his encephalopathy cleared. Due to recalcitrant alcohol abuse, he was deemed to not be a liver transplant candidate. On day 12 he had abrupt onset of severe abdominal pain and hypotension after removal of his Uldall catheter. CT of the abdomen disclosed a large retroperitoneal hematoma as seen in figure 2. He was transferred back to the ICU and treated with blood products and vasopressors but hypotension persisted. Surgical consultation was obtained but he was not felt to be a candidate for intervention.

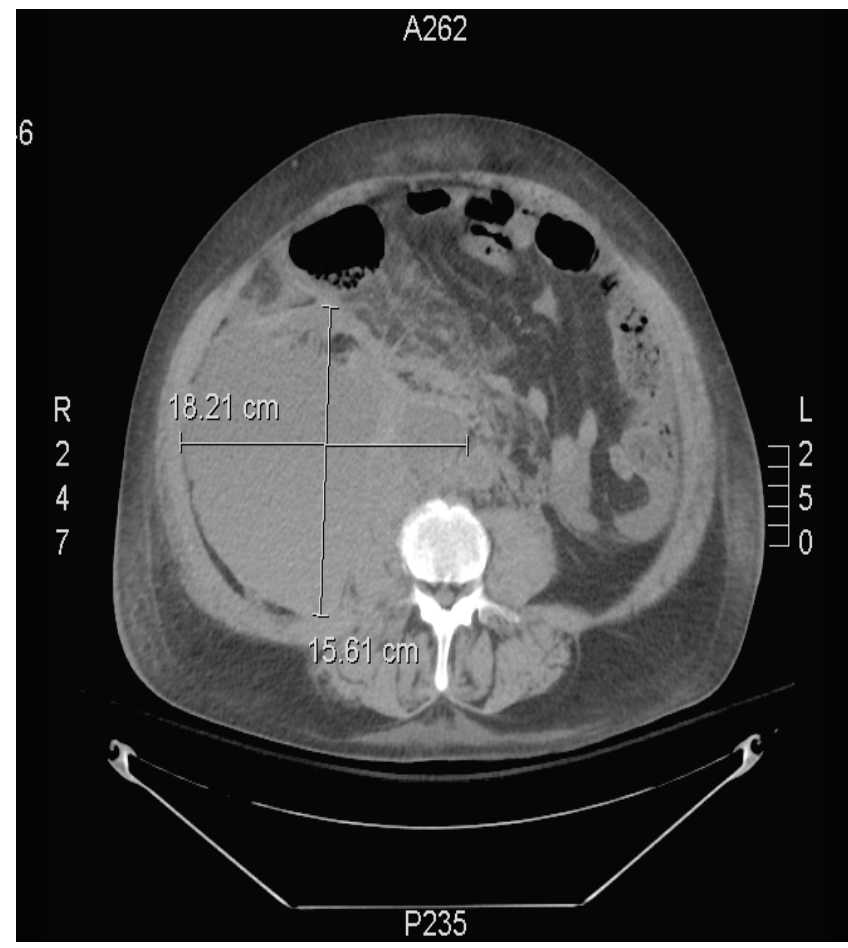

Figure 2: CT abdomen Pelvis showing large retroperitoneal Hemorrhage

The patient failed to respond to treatment for his acute retroperitoneal bleed. His MELD / MELDNa score of 46 / 45 respectively was highly predictive of mortality in the near future. A family meeting was held to discuss his poor prognosis and goals of care. The decision was made to make change his treatment to comfort measures only and he expired shortly afterward.

\section{DISCUSSION}

Comprising up to $25 \%$ of fulminant hepatic necrosis, drug toxicity is third common cause of acute liver failure behind viral and alcoholic aetiologies . ${ }^{1}$ Acetaminophen is predominantly converted to glucoronate or sulphate conjugates, which is hydrophilic and ultimately secreted in the urine. This by-product is non-toxic to the liver. When this pathway is overwhelmed by exceeding doses of acetaminophen or when levels of glutathione are depleted (glutathione is essential in detoxifying acetaminophen) the reactive intermediate, n-acetyl-p-benzoquinoneimine, accumulates and binds to intracellular macromolecules leading to apoptosis and 
centrilobular necrosis. ${ }^{2}$ Under normal circumstances, treatment of hepatotoxicity secondary to acetaminophen may be treated by $\mathrm{N}$-acetylcysteine, a precursor to glutathione, however its efficacy diminishes after 12-24-post acetaminophen exposure. ${ }^{1}$

Patients suspected of hepatic toxicity due to acute APAP are risk stratified based on the Rumack-Matthew Nomogram. This nomogram plots the patients' serum acetaminophen level against time of ingestion and is used to help determine whether a patient is a candidate for $\mathrm{N}$-acetylcysteine therapy. In patients presenting with altered mental status, unreliable history or chronic ingestion, using the nomogram for treatment decisions may be difficult. In suspected APAP ingestions, a serum level should be obtained initially and 4 hours later and the half-life should be determined. If the half-life exceeds 4 hours then the patient is a candidate for $\mathrm{N}$-acetylcysteine therapy. Serious liver injury can usually be avoided if therapy is initiated within 8 hours of ingestion. ${ }^{3}$

It has been noted that hyperbilirubinemia can interfere with commonly used assays for APAP and lead to falsely elevated serum APAP levels. In 2008, Polson et al published a study in which sera from 43 patients suffering from acute liver failure, mostly unrelated to acetaminophen, were obtained. Thirty six of the 43 patients had bilirubin concentrations ranging from 1.0-61.5 mg/dl with no acetaminophen detectable by gas chromatography mass-spectroscopy. The 36 samples were then utilized to test the performance of 2 immunoassay and 4 enzymatic colorimetric methods. Polson et al found that 3 of the 4 colorimetric methods demonstrated 'detectable' values for acetaminophen from 4 to 27 of the 36 negative samples, low concentration positive values being observed when bilirubin concentrations exceeded $10 \mathrm{mg} / \mathrm{dl}$. Polson et al concluded that false positive acetaminophen tests might result when enzymatic-colorimetric assays are used, typically when bilirubin exceeds $10 \mathrm{mg} / \mathrm{dl}^{4}{ }^{4}$ Similarly, Bertholf et al selected 12 patients without a history of acetaminophen ingestion with serum bilirubin concentrations ranging from $15-34 \mathrm{mg} / \mathrm{dl}$. They concluded that their data was consistent with bilirubin interference in the enzymatic and / or chromogenic reactions involved in the acetaminophen method, with no detectable acetaminophen levels when total bilirubin was $<5 \mathrm{mg} / \mathrm{dl}^{1}{ }^{1}$

In 2010, Roche published an urgent medical device correction regarding its assays and the risk for potential for falsely elevated levels. Based on their data, Roche determined that interference from bilirubin might produce falsely elevated values for samples containing concentrations of $30 \mu \mathrm{g} / \mathrm{ml}$ or less of acetaminophen. ${ }^{5}$ Roche's Cobas Integra 400/800 assay does advocate running serum indices on all acetaminophen samples and results must be evaluated using the appropriate serum index limits for icteric samples. Results cannot be released if it is below the measuring range or has an associated bilirubin level above the index as illustrated in table $1 .^{5}$
Table 1: An I-index of 1 corresponds to a bilirubin concentration of approximately $1 \mathrm{mg} / \mathrm{dL}$.

\begin{tabular}{cc}
\hline $\begin{array}{c}\text { Acetaminophen } \\
\text { concentration range }\end{array}$ & $\begin{array}{c}\text { Interference occurs with an } \\
\text { I index greater than }\end{array}$ \\
\hline 15 to $30 \mu \mathrm{g} / \mathrm{ml}$ & 4 \\
$>30$ to $50 \mu \mathrm{g} / \mathrm{ml}$ & 9 \\
$>50 \mu \mathrm{g} / \mathrm{ml}$ & 12 \\
\hline
\end{tabular}

Bilirubin is thought to have considerable potential for interfering with spectrophotometric measurements because of its broad, intense absorbance in the ultraviolet and visible regions of the electromagnetic spectrum. ${ }^{1}$ When definitive quantification is necessary, acetaminophen levels should be confirmed by a non-enzymatic colorimetric method such as the immunoassay techniques (EMIT and FPIA) used by Polson et al.

Since most patients with APAP overdose present before significant hepatic injury and hyperbilirubinemia have occurred, the risk of finding falsely elevated acetaminophen levels in a patient with acute overdose is usually low. It is only when the patient has significant hyperbilirubinemia that there is risk of falsely elevated APAP levels. Hyperbilirubinemia may occur in liver disease, biliary obstruction and hemolysis and consequently, treatment decisions based on APAP levels obtained in patients with these conditions may be difficult. Although treatment for APAP toxicity with $\mathrm{N}$-acetyl cysteine has low potential for harm, falsely elevated APAP levels may delay correct diagnosis and treatment. Diagnostic and therapeutic decisions based on elevated APAP levels in patients with elevated bilirubin must take into account the potential for spurious results.

\section{CONCLUSION}

Acetaminophen toxicity is a common and treatable cause of acute hepatic injury. Acetaminophen levels are an important diagnostic tool in patients with acute ingestion or hepatic injury, but may be falsely elevated in the setting of hyperbilirubinemia when commonly used assay methods are used. Falsely elevated acetaminophen levels should be considered when levels fail to decrease appropriately with time, especially in the face of appropriate treatment with $\mathrm{N}$-acetylcysteine.

When quantification is critical, clinicians should utilize immunoassay techniques, such as EMIT and FPIA, to determine accurate serum acetaminophen levels.

Falsely elevated acetaminophen levels may lead to inappropriate treatment and delay in correct diagnosis in patients with hyperbilirubinemia 
Funding- None

Conflicts of Interest - None

\section{REFERENCES}

1. Bertholf $R L$, Johganssen $L M$, Bazooband $A$, et al. False-Positive Acetaminophen Results in a Hyperbilirubinemic Patient. Clin Chem 2003;49:6958.

2. National library of Medicine. Acetaminophen [internet]. Cited; 6 Feb 2013; available at; http:// livertox.nih.gov/Acetaminophen.htm

3. Mottram AR, Kumar AM. Focus On: Acetaminophen Toxicity and Treatment [internet]. ACEP News; may
2007 [Cited 6 Feb 2013]. Available from: http://www. acep.org/content.aspx?id=26830

4. Polson J, Wians FH Jr, Orsulak P, et al. False positive acetaminophen concentrations in patients with liver injury. Clin Chim Acta 2008;391:24-30.

5. $510(k)$ Substantial equivalence determination decision summary assay only template [internet]. Available from: http://www.accessdata.fda.gov/ cdrh_docs/reviews/K110726.pdf 